\title{
Empowering women with education for better health
}

\section{U D P Ratnasiria}

(This article is based on the presidential address delivered at the induction of the $34^{\text {th }}$ President of the Sri Lanka College of Obstetricians and Gynaecologists on $5^{\text {th }}$ January 2020)

Population of Sri Lanka in 2019 was estimated as 21.7 million at 51\% of it is females (Figure 1).

$30 \%$ of the workforce is females, contributing to the country's economy according to national statistics. Their contribution ranges from manual labourer to the top-level administration and contributes immensely to the decision making at the household level to the national level. They occupy the prime role within the family as a caregiver, nurturer of values and a balanced character as a mother. The mother has the power within her to create, nurture and transform.

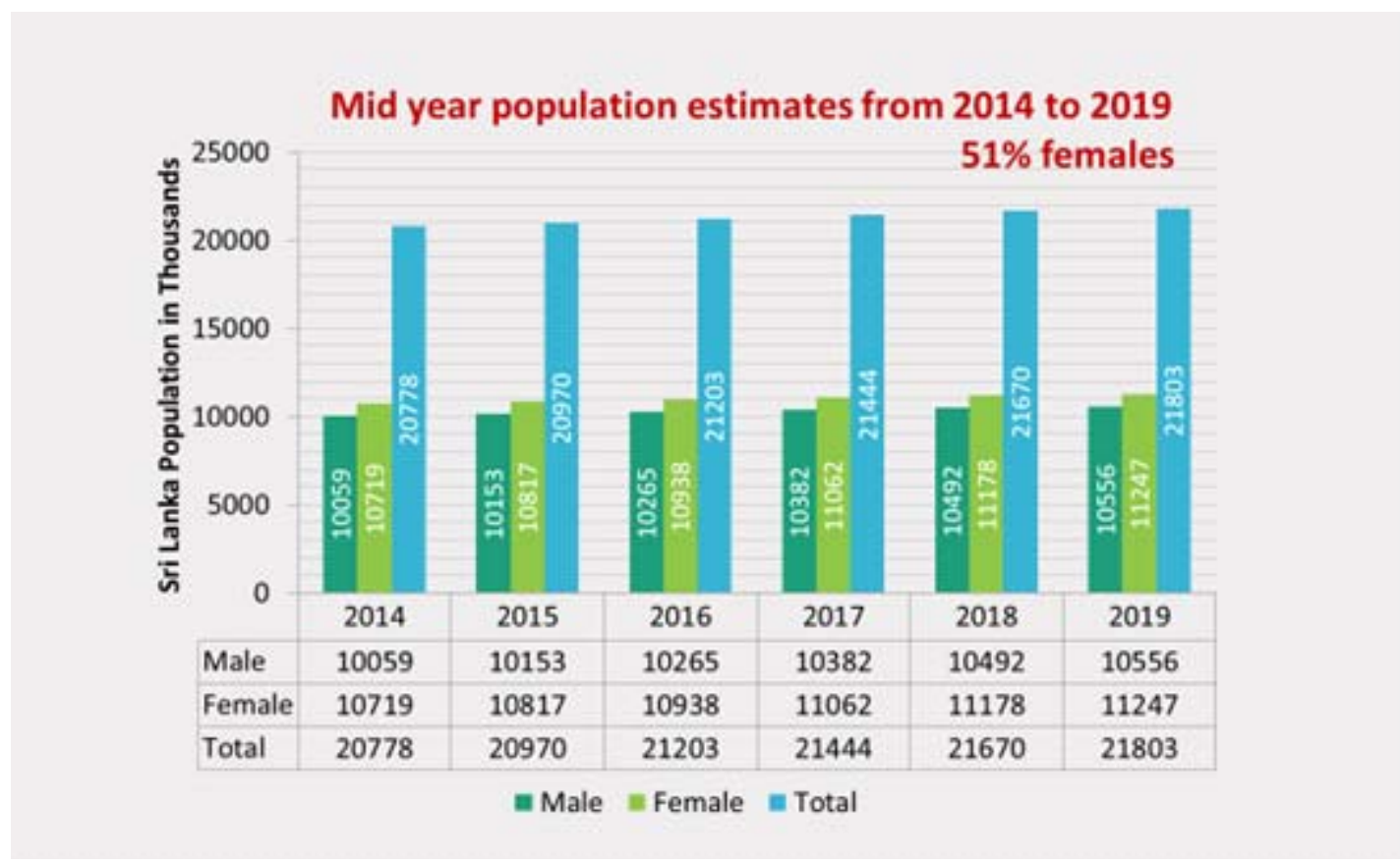

Figure 1.

Sri Lanka Journal of Obstetrics and Gynaecology 2020; 42: 2-6

DOI: http://doi.org/10.4038/sljog.v42i1.7925

a Consultant Obstetrician and Gynaecologist, Castle Street Hospital for Women, Sri Lanka.

E-mail: udpratnasiri@yahoo.com

iD http://orcid.org/0000-0002-7372-4021

This is an open-access article distributed under the terms of the Creative Commons Attribution 4.0 International License, which permits unrestricted use, distribution and reproduction in any medium provided the original author and source are credited. 
Evaluation of their contribution appears to be underestimated at all standards. Enriching women's health is an unfinished agenda, it can be converted to an achievable goal through a process of learning lessons from the history, by developing a new vision with innovative ideas and by maximizing resources. it is to understand women's health as life cycle events from conception to old age

Sri Lanka has achieved a higher level of health standards especially with maternal and perinatal morbidity and mortality in the South Asian region due to the great contribution in the past. However, these achievements are stagnated over the last two decades. The measures adapted over the past decades have brought this to current figures. The Sri Lanka College of Obstetricians and Gynaecologists understands the contribution and the achievements over the last two decades are unsatisfactory at all to any standards. Still, more than 120 pregnant women die every year and the causality changes from direct to indirect causes and many mothers die in institutions in spite of achieving 99\% institutional deliveries and excellent community maternity care. When the country achieves a low level of mortality figures further reduction requires more highly specialized care in dealing with complicated pregnancies and involvement of multidisciplinary care equally available to all the regions of the country. Analysis of maternal deaths has shown unequal distribution of facilities, sometimes even basic requirements like 24-hour blood bank services and anaesthetic cover in some parts of the island. Maldistribution of specialized care services outside obstetrics is observed in most of the mortality meetings. It has shown the poor attitude of the administration in supporting these facilities over the years.

This year my theme would be "empowering women for rightful needs of their health through transforming strategy into action”. Empowering literally means giving authority or developing self-confidence. It is a creation of an environment in which women can make independent decisions in their personal development so that they would have equal rights in society.

Health and education go hand in hand. Skill and education become the key levers for change. When women are empowered with the education, they are more effective in advocating their own health and family health.

The government and professional organizations have an obligation to ensure that these rights are met. Understanding the merits of a life-course approach to women's health is pivotal in organizing an effective care program that addresses the needs. Females face age-related physiological changes from conception to neonatal, early childhood, adolescent, reproductive and post-reproductive lives. These events are influenced by many factors such as biological, socio-cultural, nutrition and education, resulting in effecting wellbeing even in the intrauterine life. Intrauterine life can have implication not only on a girl's health during childhood but also long-term impact on the future with noncommunicable diseases which manifest usually in post-reproductive age.

The health of post-reproductive life is a culmination of a healthy conception, a healthy intrauterine life, a healthy birth, a healthy childhood, a healthy adolescent and healthy motherhood. Therefore, the challenges in front of us are enormous and complex. Sri Lanka is blessed with a strong grass root level infrastructure with a quality care distribution from the community to the hospital. It can be called as care at the doorstep by public health midwives.

Maternal and child health services are disseminated through community midwives, medical officers of health, consultant obstetrician and gynaecologists, maternity trained medical officers and nurses from the national level to the domiciliary level. Each household is designated to a public health midwife .

As the red arrow in Figure 3 indicates the mortality rate should sharply decrease by now. The time has come to investigate the reasons for failure for not achieving the necessary targets. We have been lagging behind all our neighbours in the south Asian region in the annual reduction rate it is only $6 \%$ which is alarmingly low which should be of concern to policy planners in the country. It is obvious with the current reduction rate the country will not be able to achieve SDG by 2030. If we were to achieve SGD goals of 2030, we should reduce the MMR to a figure of 13. But however, if the current trend continues our MMR will be 32 as speculated by the WHO. 

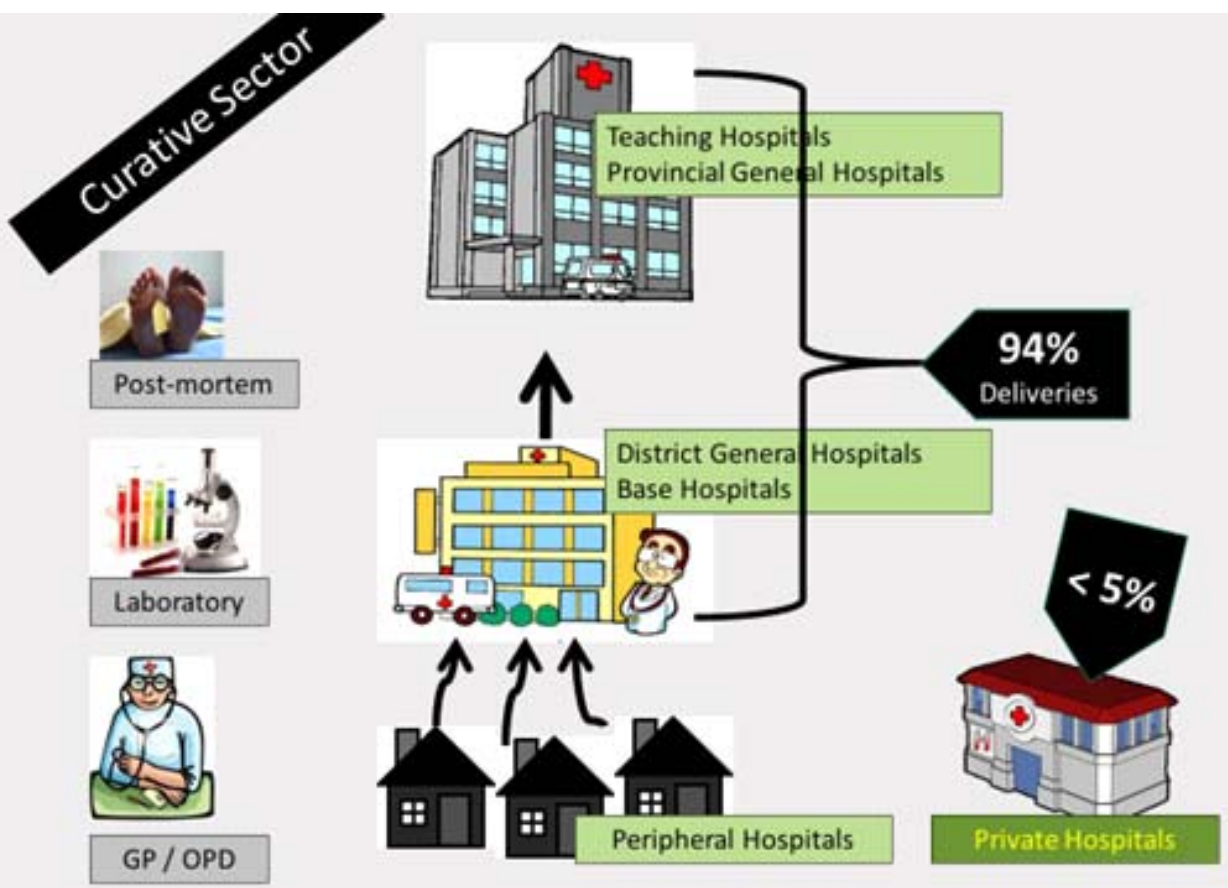

Figure 2.

\section{Progression of National MMR}

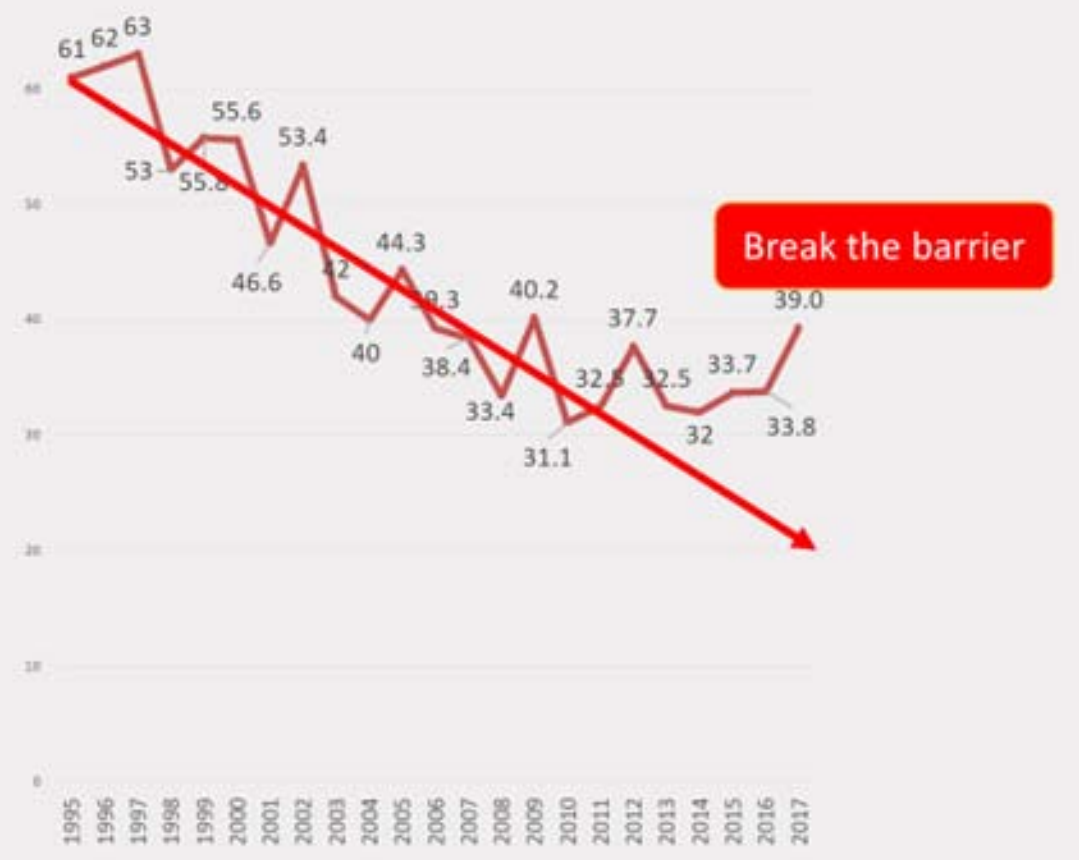

Figure 3.

Let us consider how we could overcome the present stagnation of the mortality rate. The majority of direct and indirect causes are preventable. Maternal mortality reviews have shown $69 \%$ of maternal deaths are preventable in the country with maximizing resources. 
It needs a fresh look at the entire organizational structure to develop new visions and targets. It will require a policy change, provision of new facilities, and practice of evidence-based medicine and the introduction of clinical governance systems with accountability.

I have proposed the following action plan to overcome the current situation in the country

- These action plans are based on 4 principles that would govern success.

- The government has to make women's health a national priority by the establishment of a presidential task force in women's health and provide necessary funding to strengthen the coalition. There should be greater political commitment and will.

- Establishment of a clinical governance process accountability by measuring outcome to strive for continuous improvement.

- Establishment of highly specialized units in different parts of the country and a national hospital for women.

- Changing the present system of MDSR to confidential inquiries into maternal deaths like in countries that have reached obstetric transition. In an ideal care system, preventive and curative function should function under a governance system that will monitor its quality and safety. The existing gap between the two has to be narrowed with innovative methods. This system should have a mechanism of accountability and constant auditing to identify failures and rectifying them immediately.

This would help to recognize the failures and rectify the deficiencies. The percentage of indirect deaths has surpassed the direct deaths and this information is very vital for adopting strategies to reduce the MMR.

\section{Introduction of clinical governance system}

There are different components of the clinical governance system.

This includes efficient leadership in both administrative and clinical care.
Professionals Revalidation - This will update with regard to knowledge and skills for the entire team.

Clinical Risk Management-Any danger to the function of the system will be identified promptly.

The patient's suggestions and complaints will help to make the system more efficient and patient-friendly.

Evidence-based Guidelines of Education and Training Audit - The system will run according to the standard current excepted guidelines. The system will undergo constant auditing to identify system failures.

Accredited Services - These services are the blood bank and labs, intensive care, high dependency units, operating theatres, transport, communication, and a referral system.

This entire system has to be funded by the government.

\section{Introduction of maternity dashboard to all delivery units}

This is to assess important parameters in the delivery unit with a comparable assessment with set standards using colour codes on a monthly basis.

This includes workforce, activity and clinical indicators. Eg. Staffing level, caesarean section, instrumental delivery rates, maternal and perinatal mortality rates. This is an alarm system is called the maternity dashboard. When the system is failing a red signal appears in the dashboard.

Amber color indicates a warning message its needs help.

If the maternity dashboard is applied to a labour room red color signal indicates immediate attention to bring into the green.

\section{Establishment of highly specialized centers}

Indirect causes leading to maternal deaths are escalating in the country. Many of these patients need multidisciplinary care with close monitoring in highly specialized units. 
In view of the increasing contribution of medical disorders such as cardiovascular, respiratory, liver and renal failure secondary to obstetric emergencies availability of advanced treatment centers should be established to manage seriously ill patients. On the recommendation of the action plan government is providing financial assistance to establish such units in Colombo, Kandy, Galle and Anuradhapura. This will provide a state of the art multi-disciplinary care for needy patients.

It is disheartening to note that this project was initiated by the government on the recommendation of SLCOG in 2014 and fail to complete. The SLCOG earnestly requests the government to allocate funding for this purpose and complete these projects soon.

\section{Establishment of the National Taskforce}

SLCOG proposed to establish a national task force in sexual and reproductive health. This has to bring all stakeholders under one umbrella.

It will enable national planning, implementation, monitoring and evaluation by a central body. Institutional clinical governance processes can be linked through a provincial process with the Centre for Monitoring and Evaluation.

Incorporating the Attorney General Department into the national task force will greatly help to overcome the legislative limitations we encounter in maternal deaths and adolescent issues such as rape and teenage pregnancy. College is hoping to establish a discussion with the relevant authorities of the Health Ministry for implementation.

\section{Establishing confidential inquiry into maternal deaths}

Currently in Sri Lanka maternal deaths are reviewed in an open forum with all stakeholders. The SLCOG had already drafted a document to propose a confidential review for maternal deaths. This system will bring about confidentiality and could solve many issues related to present system and the national recommendation would address the deficiencies which could have contributed to maternal deaths.

\section{Concerns about increase cesarean section rates}

Increasing cesarean section rates have been observed over the last two decades and currently, the number reaches 39.1\% in 2017. This has brought the rising incidence of placental adhesive disorders which could lead to more maternal morbidity and mortality.

Application of the Robson criteria and identifying and analyzing the highest groups and clinical approaches to reduce the numbers.

Minimizing inductions of labour for uncomplicated pregnancies and giving them the chance to start naturally can reduce many inductions and so primary caesareans.

Clinical interventions, such as external cephalic version for breech presentation at term, vaginal breech delivery in appropriately selected women, and vaginal birth after $\mathrm{CS}$, could reduce the frequency of CS use.

\section{Conducting continuous professional develop- ment programs for health care workers}

Education, training and skill development are integral components of an effective clinical governance process. Members of SLCOG have voluntarily taken the responsibility as team leaders to engage in this activity.

The College conducts frequent safe motherhood programs to update the knowledge and skills of junior doctors, maternity care nurses and midwives in emergency obstetrics.

I believe all the above-mentioned activities would help to improve the quality of care given to women in the country resulting in a further reduction in mortality and morbidity. I anticipate cordial cooperation and support of the fellows and members of SLCOG to achieve these targets in the current year. 